\title{
The Hankel transform of generalized central trinomial coefficients and related sequences
}

\author{
Marko D. Petković ${ }^{1}$, Predrag M. Rajković ${ }^{2}$, Paul Barry ${ }^{3}$ \\ ${ }^{1}$ Faculty of Science and Mathematics, University of Niš \\ Višegradska 33, 18000 Niš, Serbia \\ E-mail: dexterofnis@gmail.com \\ 2 Faculty of Mechanical Engineering, University of Niš \\ Aleksandra Medvedeva 14, 18000 Niš, Serbia \\ E-mail: pedja.rajk@yahoo.com \\ ${ }^{3}$ School of Science, Waterford Institute of Technology, Ireland \\ E-mail: pbarry@wit.ie
}

\begin{abstract}
In this paper, we explore the connection between the Hankel trasform, Riordan arrays and orthogonal polynomials. For this purpose, we evaluate the Hankel transform of generalized trinomial coefficients, as a closed-form expression, using the method based on the orthogonal polynomials. Since the generalized trinomial coefficients are generalization of several integer sequences, obtained expression is also applicable in these cases. We also showed that the coefficient array of corresponding orthogonal polynomials can be represented in terms of Riordan arrays, which provides the $L D L^{T}$ decomposition of the Hankel matrix. Moreover we consider the row sums of the inverse of coefficient array matrix and evaluate its Hankel transform.
\end{abstract}

MS Classification: Primary 11B83; Secondary 05A19, 33C45, $11 \mathrm{~B} 37$.

Keywords: Hankel transform, Orthogonal polynomials, Central trinomial coefficients, Riordan arrays.

\section{Introduction}

In this paper we take the generalized central trinomial numbers [13] as a vehicle to explore the links that exist between certain sequences of integers, orthogonal polynomials, Riordan arrays and Hankel transform. In order to make this paper reasonably self-contained, we give a brief introduction to each of these topics in this section.

We shall use the notation $a_{n}$ to denote the general term of the integer sequence $\left(a_{n}\right)_{n \in \mathbb{N}_{0}}$ where $a_{n} \in \mathbb{Z}$. The ordinary generating function of the sequence $a_{n}$ is the power series $f(x)=$ $\sum_{n=0}^{+\infty} a_{n} x^{n}$. We also denote $a_{n}=\left[x^{n}\right] f(x)$, i.e. the operator $\left[x^{n}\right]$ extracts the coefficient of $x^{n}$ in the power series expansion of $f(x)$. Sequences are often referred to by their 'A' number in the On-Line Encyclopedia of Integer Sequences [16, 17].

Example 1. The central binomial coefficients sequence $\left(b_{n}\right)_{n \in \mathbb{N}_{0}}$ has a general term $b_{n}=\left(\begin{array}{c}2 n \\ n\end{array}\right)$. It is referred as A000984 in the On-Line Encyclopedia and has first few members equal to $(1,2,6,20,70,252, \ldots)$. The generating function of this sequence is $\frac{1}{\sqrt{1-4 x}}$. 
The study of integer sequences often involves looking at transformations that send one integer sequence into another one. Linear transformations are characterized by infinite matrices. If we regard the sequence $\left(a_{n}\right)_{n \in \mathbb{N}_{0}}$ as a column vector $\left[\begin{array}{lll}a_{0} & a_{1} \cdots\end{array}\right]^{T}$, every linear transformation can be written as $b=\mathbf{M} a$, where $\mathbf{M}$ is an infinite matrix. We use that matrix notation in the rest of the paper. Since the sequences start from index 0, we take the top left term of the matrix $\mathbf{M}$ to have an index $(0,0)$.

Definition 1. The binomial transform of the sequence $\left(a_{n}\right)_{n \in \mathbb{N}_{0}}$ returns the sequence $\left(b_{n}\right)_{n \in \mathbb{N}_{0}}$ defined by (see [20]) $b_{n}=\sum_{k=0}^{n}\left(\begin{array}{l}n \\ k\end{array}\right) a_{k}$. This transformation is invertible, with inversion formula $a_{n}=\sum_{k=0}(-1)^{n-k}\left(\begin{array}{l}n \\ k\end{array}\right) b_{k}$. Binomial transformation can be represented by the matrix

$$
\mathbf{B}=\left(\begin{array}{ccccc}
1 & 0 & 0 & 0 & \ldots \\
1 & 1 & 0 & 0 & \\
1 & 2 & 1 & 0 & \\
1 & 3 & 3 & 1 & \\
\vdots & & & & \ddots
\end{array}\right)
$$

with general term $\left(\begin{array}{l}n \\ k\end{array}\right)$.

A special class of infinite matrices, called Riordan arrays, is very useful in the study of linear matrix transformations.

Definition 2. The Riordan array is an infinite lower-triangular matrix $\mathbf{M}=\left(m_{j, k}\right)_{j, k \in \mathbb{N}_{0}}$ defined by a pair of generating functions $g(x)=\sum_{k=0}^{+\infty} g_{k} x^{k}$ and $f(x)=\sum_{k=1}^{+\infty} f_{k} x^{k}$ where $f_{1} \neq 0$, whose $k$-th column is generated by $g(x) f(x)^{k}$ (the first column being indexed by 0 ), i.e.

$$
m_{j, k}=\left[x^{j}\right]\left(g(x) f(x)^{k}\right) \quad\left(j, k \in \mathbb{N}_{0}\right) .
$$

The matrix $\mathbf{M}$ corresponding to the pair of functions $f$ and $g$ is denoted by $(g, f)$ or $\mathcal{R}(g, f)$.

Note that the set of Riordan arrays is closed under the matrix multiplication and matrix inversion and therefore have the structure of group (see [15] and [18]).

Lemma 1. $[15,18]$ Let $(g, f)$ and $(h, l)$ are Riordan arrays. Their matrix product is given by $(g, f) \cdot(h, l)=(g \cdot(h \circ f), l \circ f)$, where $\circ$ denotes the composition of functions. The identity and the inverse of $(g, f)$ are given by $I=(1, x)$ and $(g, f)^{-1}=\left(\frac{1}{g \circ f}, \bar{f}\right)$, where $\bar{f}$ denotes the compositional inverse of $f$ in the neighborhood of the point $x=0$.

It is worth mentioning that, according to the Inverse Function Theorem, function $f(x)$ is invertible in the neighborhood of the point $x=0$ since $f^{\prime}(0)=f_{1} \neq 0$. Moreover, its inverse $\bar{f}(x)$ can also be represented as the power sequence $\bar{f}(x)=\sum_{n=1}^{+\infty} u_{n} x^{n}$ where $\bar{f}^{\prime}(x)=u_{1} \neq 0$.

The following theorem connects the generating functions of the row sums and diagonal of the Riordan array $\mathbf{M}=(g, f)$.

Lemma 2. $[15,18]$ Let $\mathbf{M}=\left(m_{n, j}\right)_{n, j \in \mathbb{N}_{0}}=(g, f)$ is the Riordan array. It is valid

$$
\sum_{i=0}^{+\infty}\left(\sum_{j=0}^{\infty} m_{i, j}\right) x^{i}=\frac{g(x)}{1-f(x)}, \quad \sum_{i=0}^{\infty} m_{i, i} x^{i}=\frac{g(x)}{1-x f(x)}
$$

Example 2. The binomial matrix $\mathbf{B}$ is Riordan array $\left(\frac{1}{1-x}, \frac{x}{1-x}\right)$. More generally, $\mathbf{B}^{m}$ is Riordan array $\left(\frac{1}{1-m x}, \frac{x}{1-m x}\right)$, with general term $\left(\begin{array}{l}n \\ k\end{array}\right) m^{n-k}$. It is easy to show that the inverse $\mathbf{B}^{-m}$ of $\mathbf{B}^{m}$ is given by $\left(\frac{1}{1+m x}, \frac{x}{1+m x}\right)$. 
We have seen that the binomial transform is invertible. An important transform on integer sequences that has been much studied recently, which is not invertible, is the Hankel transform $[3,12,14]$

Definition 3. The Hankel transform of a given sequence $a=\left(a_{n}\right)_{n \in \mathbb{N}_{0}}$ is the sequence of Hankel determinants $\left(h_{n}\right)_{n \in \mathbb{N}_{0}}$ where $h_{n}=\operatorname{det}\left[a_{i+j-2}\right]_{i, j=1}^{n}$, i.e

$$
a=\left(a_{n}\right)_{n \in \mathbb{N}_{0}} \quad \Longrightarrow^{\mathcal{H}} \quad h=\left(h_{n}\right)_{n \in \mathbb{N}_{0}}: \quad h_{n}=\operatorname{det}\left[\begin{array}{cccc}
a_{0} & a_{1} & \cdots & a_{n} \\
a_{1} & a_{2} & & a_{n+1} \\
\vdots & & \ddots & \\
a_{n} & a_{n+1} & & a_{2 n}
\end{array}\right]
$$

We denote Hankel transform by $\mathcal{H}$ and hence we write $h=\mathcal{H}(a)$.

Hankel determinants are sometimes also called persymmetric or Turanian determinants. Although the determinants of Hankel matrices had been studied before, the term Hankel transform was introduced in 2001 by Layman [12]. In that paper it is also proven that the Hankel transform is invariant under the Binomial transform.

Lemma 3. (Layman 2001) [12] The Hankel transform of a sequence $\left(a_{n}\right)_{n \in \mathbb{N}_{0}}$ and Hankel transform of its binomial transform are equal.

Many different evaluations of the Hankel transform are known in the literature. Brualdi and Kirkland [1] evaluated the Hankel transform of large Schroder numbers using a method based on continued fractions. In [8], there are shown several evaluations of the Hankel transform based on the exponential generating function. Recently in [19], Xin proved that the Hankel transform of the certain number sequence is the Somos-4 sequence. M.E.H. Ismail evaluated several Hankel determinants with orthogonal polynomial entries in [7]. Moreover, Egecioglu, Redmond and Ryavec $[4,5]$ considered a method based on differential-convolution equations which is useful when the Hankel transform can be written as the sum of a small number of products (almost product). Also, classic methods for determinant evaluation like Dodgson condenzation and LU decomposition method are applicable in some cases of the Hankel determinants. A survey of different methods for determinant evaluations, including Hankel determinants, is given in the papers of Krattenthaler [9, 10].

In this paper, we use the method based on the orthogonal polynomials for a Hankel transform evaluation. That method was already used by Cvetković, Rajković and Ivković for computing the Hankel transform of the sequence whose general term is the sum of two consecutive Catalan numbers [3]. Present authors provided the generalization of the result in [3] and evaluated the Hankel transform of sums of two consecutive generalized Catalan numbers [14]. Here we briefly restate that method.

Let $\left(a_{n}\right)_{n \in \mathbb{N}_{0}}$ be the moment sequence with respect to some measure $d \lambda$. In other words, let

$$
a_{n}=\int_{\mathbb{R}} x^{n} d \lambda \quad(n=0,1,2, \ldots) .
$$

Then the Hankel transform $h=\mathcal{H}(a)$ of the sequence $a=\left(a_{n}\right)_{n \in \mathbb{N}_{0}}$ can be expressed by the following relation known as Heilermann formula (for example, see Krattenthaler [10])

$$
h_{n}=a_{0}^{n+1} \beta_{1}^{n} \beta_{2}^{n-1} \cdots \beta_{n-1}^{2} \beta_{n} .
$$

Sequence $\left(\beta_{n}\right)_{n \in \mathbb{N}_{0}}$ is the sequence of coefficients of the three-term recurrence relation

$$
P_{n+1}(x)=\left(x-\alpha_{n}\right) P_{n}(x)-\beta_{n} P_{n-1}(x),
$$


satisfied by the sequence $\left(P_{n}(x)\right)_{n \in \mathbb{N}_{0}}$ of monic orthogonal polynomials with respect to the measure $d \lambda$.

The following theorem and corollary provide the way how to explicitly find the measure $d \lambda$ with prescribed moment sequence.

Theorem 4. (Stieltjes-Perron inversion formula) $[2,11]$ Let $\left(\mu_{n}\right)_{n \in \mathbb{N}_{0}}$ is the sequence such that all elements of its Hankel transform are non-negative. Denote by $G(z)=\sum_{n=0}^{+\infty} \mu_{n} z^{n}$ the generating function of the sequence $\left(\mu_{n}\right)_{n \in \mathbb{N}_{0}}$ and $F(z)=z^{-1} G\left(z^{-1}\right)$. Also let the function $\lambda(t)$ is defined by

$$
\lambda(t)-\lambda(0)=-\frac{1}{2 \pi i} \lim _{y \rightarrow 0^{+}} \int_{0}^{t}[F(x+i y)-F(x-i y)] d x .
$$

Then holds $\mu_{n}=\int_{\mathbb{R}} x^{n} d \lambda$, i.e. sequence $\left(\mu_{n}\right)_{n \in \mathbb{N}_{0}}$ is the moment sequence of the measure $\lambda(t)$.

Corollary 5. Under the assumptions of the previous lemma, let additionally holds $F(\bar{z})=\overline{F(z)}$. Then

$$
\lambda(t)-\lambda(0)=-\frac{1}{\pi} \lim _{y \rightarrow 0^{+}} \int_{0}^{t} \Im F(x+i y) d x .
$$

This paper is organized as follows. In Section 2, we recall the definition and basic properties of the sequence of generalized central trinomial coefficients. In Section 3, we give a closed-form expression of the Hankel transform of generalized central trinomial coefficients. After that, we show that the coefficient array of corresponding orthogonal polynomials can be expressed as Riordan array. Finally we provide the Hankel transform evaluation of the row sums of inverse coefficient array.

The first Hankel transform evaluation has product form and hence, the method based on orthogonal polynomials can be directly applied. This is not the case with the second evaluation where we have to modify our approach and to use difference equations and generating functions.

All presented results show that there is a connection between Hankel transform, Riordan arrays and orthogonal polynomials and provide a new way how to deal with the non-product Hankel transform evaluation (different to one shown in $[4,5]$ ).

\section{Generalized central trinomial coefficients}

For a given integers $a, b, c \in \mathbb{N}$, coefficient of $x^{n}$ in the expression $\left(a+b x+c x^{2}\right)^{n}$ is known as generalized central trinomial coefficient. A term generalized central trinomial coefficient is first introduced by Noe in the paper [13]. The following summation formula is obtained by applying binomial theorem twice [13]:

$$
\left[x^{n}\right]\left(a+b x+c x^{2}\right)^{n}=\sum_{k=0}^{\left\lfloor\frac{n}{2}\right\rfloor}\left(\begin{array}{c}
2 k \\
k
\end{array}\right)\left(\begin{array}{c}
n \\
2 k
\end{array}\right) b^{n-2 k}(a c)^{k}
$$

It can be seen that previous expression depends on $a$ and $c$ only by its product $a c$. Therefore, without loosing of generality we can assume that $a=1$, i.e. that generalized central trinomial coefficients are defined by

$$
t_{n}(\alpha, \beta)=\left[x^{n}\right]\left(1+\alpha x+\beta x^{2}\right)^{n}=\sum_{k=0}^{\left\lfloor\frac{n}{2}\right\rfloor}\left(\begin{array}{c}
2 k \\
k
\end{array}\right)\left(\begin{array}{c}
n \\
2 k
\end{array}\right) \alpha^{n-2 k} \beta^{k} .
$$


First few members of the sequence $\left(t_{n}(\alpha, \beta)\right)_{n \in \mathbb{N}_{0}}$ are $\left(1, \alpha, \alpha^{2}+2 \beta, \alpha^{3}+6 \alpha \beta, \alpha^{4}+12 \alpha^{2} \beta+\right.$ $\left.6 \beta^{2}, \alpha^{5}+20 \alpha^{3} \beta+30 \alpha \beta^{2}, \ldots\right)$.

In the paper [13], there are shown many properties and representations of the sequence $\left(t_{n}(\alpha, \beta)\right)_{n \in \mathbb{N}_{0}}$ including an ordinary generating function, exponential generating function, representation via Legendre polynomials, divisibility properties, etc. It is also proven the following result concerning the generating function $T(x)$ of generalized central trinomial coefficients which will be useful in the next section.

Theorem 6. (Noe 2006) [13] The generating function for $t_{n}(\alpha, \beta)$ is given by

$$
T(x)=\frac{1}{\sqrt{1-2 \alpha x+\left(\alpha^{2}-4 \beta\right) x^{2}}} .
$$

Sequence $\left(t_{n}(\alpha, \beta)\right)_{n \in \mathbb{N}_{0}}$ provides the generalization of the many known integer sequences (see table in the appendix of [13]). We mention three most important generalizations:

- Central trinomial coefficients (A002426) defined by $t_{n}=\left[x^{n}\right]\left(1+x+x^{2}\right)^{n}=t_{n}(1,1)$. First few members of this sequence are $(1,1,3,7,19,51, \ldots)$ while generating function is $\frac{1}{\sqrt{1-2 x-3 x^{2}}}$.

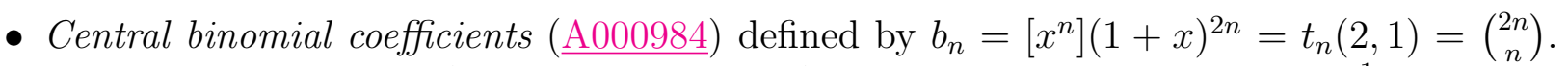
First few members are $(1,2,6,20,70,252, \ldots)$ while generating function is $\frac{1}{\sqrt{1-4 x}}$.

- Aerated central binomial coefficients defined by $e_{n}=\left[x^{n}\right]\left(1+x^{2}\right)^{n}=t_{n}(0,1)$. This sequence has zeros on odd positions, while the general term on even position $2 k$ is central binomial coefficient $\left(\begin{array}{c}2 k \\ k\end{array}\right)$. First few members are $(1,0,2,0,6,0,20, \ldots)$ while generating function is $\frac{1}{\sqrt{1-4 x^{2}}}$.

Therefore, results concerning the Hankel transform of the sequence $\left(t_{n}(\alpha, \beta)\right)_{n \in \mathbb{N}_{0}}$, shown in the following section, can be also applied to the sequences mentioned above and also to other sequences which generalization is $\left(t_{n}(\alpha, \beta)\right)_{n \in \mathbb{N}_{0}}$.

\section{The Hankel transform of generalized central trinomial coefficients}

In this section, we evaluate the Hankel transform of the sequence of generalized central trinomial coefficients. Moreover we provide a few more properties of corresponding orthogonal polynomials and their coefficient array.

In the rest of this section we assume that $\beta>0$. Since the obtained expression for the Hankel transform of $\left(t_{n}(\alpha, \beta)\right)_{n \in \mathbb{N}_{0}}$ is polynomial as a function of $\beta$, it holds for arbitrary $\beta$. The following theorem which gives an explicit expression of the weight function whose moment sequence is $\left(t_{n}(\alpha, \beta)\right)_{n \in \mathbb{N}_{0}}$.

Theorem 7. Generalized central trinomial coefficients $t_{n}(\alpha, \beta)$ are moments of the weight function

$$
w(x)=\left\{\begin{array}{ll}
\frac{1}{\pi} \frac{1}{\sqrt{4 \beta-(x-\alpha)^{2}}}, & x \in[\alpha-2 \sqrt{\beta}, \alpha+2 \sqrt{\beta}] \\
0, & \text { otherwise }
\end{array} .\right.
$$


Proof. The proof is based on the Stieltjes-Perron inversion formula (Theorem 4). We start from

$$
F(z)=z^{-1} T\left(z^{-1}\right)=\frac{1}{\sqrt{z^{2}-2 \alpha z+\alpha^{2}-4 \beta^{2}}}=\frac{1}{\sqrt{\left(z-x_{1}\right)\left(z-x_{2}\right)}},
$$

where $x_{1,2}=\alpha \pm 2 \sqrt{\beta}$ are branch points of the function $F(z)$. We take a regular branch of $F(z)$ such that $\arg \left(z-x_{1}\right)=\arg \left(z-x_{2}\right)=0$ for $z \in\left(x_{2},+\infty\right)$. Selected branch is defined on $\mathbb{C} \backslash\left(x_{1}, x_{2}\right)$ and satisfies $\overline{F(z)}=F(\bar{z})$.

By direct evaluation we find the primitive function of $F(z)$ :

$$
F_{1}(z)=\int F(z) d z=\log 2+\log \left(z-\alpha+\sqrt{(z-\alpha)^{2}-4 \beta}\right) .
$$

We take a regular branch of $\log$ function on the set $\mathbb{C} \backslash[0,+\infty)$ such that $\lim _{y \rightarrow 0+} \log (x+i y)=$ $\log x$ when $x>0$. Now using

$$
\lim _{y \rightarrow 0} \Im \sqrt{(x+i y-\alpha)^{2}-4 \beta}=\left\{\begin{array}{ll}
\sqrt{4 \beta-(x-\alpha)^{2}}, & x \in\left(x_{1}, x_{2}\right) \\
0, & \text { otherwise }
\end{array} .\right.
$$

we obtain

$$
G(x)=\lim _{y \rightarrow 0} \Im F_{1}(x+i y)= \begin{cases}\pi, & x<x_{1} \\ \pi+\arctan \left(\frac{\sqrt{4 \beta-(x-\alpha)^{2}}}{x-\alpha}\right), & x \in\left[x_{1}, \alpha\right) \\ \frac{\pi}{2}, & x=\alpha \\ \arctan \left(\frac{\sqrt{4 \beta-(x-\alpha)^{2}}}{x-\alpha}\right), & x \in\left(\alpha, x_{2}\right] \\ 0, & x>x_{2}\end{cases}
$$

Now from Corollary 5 directly yields $\lambda(t)=-\frac{1}{\pi}(G(t)-G(0))$. Since $d \lambda$ is absolutely continuous measure, we obtain the expression (9) by differentiation of $\lambda(t)$. This completes the proof of the theorem.

Previous theorem implies the following integral representation of generalized central trinomial coefficients

$$
t_{n}(\alpha, \beta)=\frac{1}{\pi} \int_{\alpha-2 \sqrt{\beta}}^{\alpha+2 \sqrt{\beta}} \frac{x^{n}}{\sqrt{\left(4 \beta-\alpha^{2}\right)+2 \alpha x-x^{2}}} d x=\frac{1}{\pi} \int_{\alpha-2 \sqrt{\beta}}^{\alpha+2 \sqrt{\beta}} \frac{x^{n}}{\sqrt{4 \beta-(x-\alpha)^{2}}} d x .
$$

Note that for $\alpha=2$ and $\beta=1$, Theorem 7 implies the following well-known moment representation of central binomial coefficients $[16,17]$

$$
t_{n}(2,1)=\left(\begin{array}{c}
2 n \\
n
\end{array}\right)=\frac{1}{\pi} \int_{0}^{4} \frac{x^{n}}{\sqrt{x(4-x)}} d x .
$$

Similarly holds for the aerated central binomial coefficients and central trinomial coefficients

$$
\begin{aligned}
& t_{n}(0,1)=\frac{1+(-1)^{n}}{2}\left(\begin{array}{c}
2 n \\
n
\end{array}\right)=\frac{1}{\pi} \int_{-2}^{2} \frac{x^{n}}{\sqrt{4-x^{2}}} d x, \\
& t_{n}(1,1)=\frac{1}{\pi} \int_{-1}^{3} \frac{x^{n}}{\sqrt{3+2 x-x^{2}}} d x .
\end{aligned}
$$

One method for determining coefficients of three-term recurrence relation corresponding to some weight function is to apply transformation formulas. These formulas connect the coefficients $\alpha_{n}$ and $\beta_{n}$ of the original and transformed weight function. The following lemma gives the transformation formulas for two different transformations. 
Lemma 8. Let $w(x)$ and $\tilde{w}(x)$ be weight functions and denote by $\left(\pi_{n}(x)\right)_{n \in \mathbb{N}_{0}}$ and $\left(\tilde{\pi}_{n}(x)\right)_{n \in \mathbb{N}_{0}}$ corresponding orthogonal polynomials. Also denote by $\left(\alpha_{n}\right)_{n \in \mathbb{N}_{0}},\left(\beta_{n}\right)_{n \in \mathbb{N}_{0}}$ and $\left(\tilde{\alpha}_{n}\right)_{n \in \mathbb{N}_{0}},\left(\tilde{\beta}_{n}\right)_{n \in \mathbb{N}_{0}}$ three-term relation coefficients corresponding to $w(x)$ and $\tilde{w}(x)$ respectively. The following transformation formulas are valid:

(1) If $\tilde{w}(x)=C w(x)$ where $C>0$ then holds $\tilde{\alpha}_{n}=\alpha_{n}$ for $n \in \mathbb{N}_{0}$ and $\tilde{\beta}_{0}=C \beta_{0}, \tilde{\beta}_{n}=\beta_{n}$ for $n \in \mathbb{N}$. Additionally holds $\tilde{\pi}_{n}(x)=\pi_{n}(x)$ for all $n \in \mathbb{N}_{0}$.

(2) If $\tilde{w}(x)=w(a x+b)$ where $a, b \in \mathbb{R}$ and $a \neq 0$ there holds $\tilde{\alpha}_{n}=\frac{\alpha_{n}-b}{a}$ for $n \in \mathbb{N}_{0}$ and $\tilde{\beta}_{0}=\frac{\beta_{0}}{|a|}$ and $\tilde{\beta}_{n}=\frac{\beta_{n}}{a^{2}}$ for $n \in \mathbb{N}$. Additionally holds $\tilde{\pi}_{n}(x)=\frac{1}{a^{n}} \pi_{n}(a x+b)$.

Proof. In both cases, we directly check the orthogonality of $\tilde{\pi}_{n}(x)$ and obtain the coefficients $\tilde{\alpha}_{n}$ and $\tilde{\beta}_{n}$ by putting $\tilde{\pi}_{n}(x)$ in the three-term recurrence relation for $\pi_{n}(x)$.

Now we are ready to calculate the Hankel transform the sequence $\left(t_{n}(\alpha, \beta)\right)_{n \in \mathbb{N}_{0}}$.

Theorem 9. The Hankel transform of the sequence $\left(t_{n}(\alpha, \beta)\right)_{n \in \mathbb{N}_{0}}$ is given by $h_{n}(\alpha, \beta)=$ $2^{n} \beta^{\left(\begin{array}{c}n+1 \\ 2\end{array}\right)}$.

Proof. We use Heilermann formula (5) and weight function transformation given by Lemma 8. Since

$$
w(x)= \begin{cases}\frac{1}{\pi} \frac{1}{\sqrt{4 \beta-(x-\alpha)^{2}}}, & x \in[\alpha-2 \sqrt{\beta}, \alpha+2 \sqrt{\beta}] \\ 0, & \text { otherwise }\end{cases}
$$

we start from the monic Chebyshev polynomials of the first kind $\hat{T}_{n}(x)$ and their weight function $w_{0}(x)$ given by [2]:

$$
w_{0}(x)=\left\{\begin{array}{ll}
\frac{1}{\sqrt{1-x^{2}}}, & x \in[-1,1] \\
0, & \text { otherwise }
\end{array} .\right.
$$

Coefficients $\alpha_{n}^{(0)}$ and $\beta_{n}^{(0)}$ corresponding to $w_{0}(x)$ are equal to [2]

$$
\alpha_{n}^{(0)}=0, \quad \beta_{0}^{(0)}=\pi, \quad \beta_{1}^{(0)}=\frac{1}{2}, \quad \beta_{n}^{(0)}=\frac{1}{4}, \quad(n>1) .
$$

Now since

$$
w_{1}(x)=\frac{1}{\sqrt{4 \beta-(x-\alpha)^{2}}}=\frac{1}{2 \sqrt{\beta}} \frac{1}{\sqrt{1-\left(\frac{x-\alpha}{2 \sqrt{\beta}}\right)^{2}}}=\frac{1}{2 \sqrt{\beta}} w_{0}\left(\frac{x-\alpha}{2 \sqrt{\beta}}\right) .
$$

we can use part b) of Lemma 8 where $a=1 /(2 \sqrt{\beta})$ and $b=-\alpha /(2 \sqrt{\beta})$. Hence we obtain

$$
\alpha_{n}^{(1)}=\alpha \quad(n \in \mathbb{N}), \quad \beta_{0}^{(1)}=\pi, \quad \beta_{1}^{(1)}=2 \beta, \quad \beta_{n}^{(1)}=\beta \quad(n=2,3, \ldots) .
$$

Moreover, monic orthogonal polynomials corresponding to $w_{1}(x)$ are given by

$$
P_{n}^{(1)}(x)=(2 \sqrt{\beta})^{n} \hat{T}_{n}\left(\frac{x-\alpha}{2 \sqrt{\beta}}\right) .
$$

Now let $w(x)=\frac{1}{\pi} w_{1}(x)$. From part a) of the Lemma 8 we see that $\alpha_{n}=\alpha_{n}^{(1)}=1$ for every $n \in \mathbb{N}_{0}$. Also $\beta_{1}=\beta_{1}^{(1)}=2 \beta$ and $\beta_{n}=\beta_{n}^{(1)}=\beta$ while $\beta_{0}=\frac{1}{\pi} \cdot \beta_{0}^{(1)}=1$. Hence

$$
\alpha_{n}=\alpha \quad(n \in \mathbb{N}), \quad \beta_{0}=1, \quad \beta_{1}=2 \beta, \quad \beta_{n}=\beta \quad(n=2,3, \ldots) .
$$


Note that the family of monic orthogonal polynomials $P_{n}(x)$ associated to the weight $w(x)$ are

$$
P_{n}(x)=P_{n}^{(1)}(x)=(2 \sqrt{\beta})^{n} \hat{T}_{n}\left(\frac{x-\alpha}{2 \sqrt{\beta}}\right) .
$$

Applying Heilermann formula (5) we finally obtain the closed-form expression for the Hankel transform $\left(h_{n}(\alpha, \beta)\right)_{n \in \mathbb{N}_{0}}$ of the sequence $\left(t_{n}(\alpha, \beta)\right)_{n \in \mathbb{N}_{0}}$ :

$$
h_{n}(\alpha, \beta)=t_{0}(\alpha, \beta)^{n+1} \beta_{1}^{n} \beta_{2}^{n-1} \cdots \beta_{n-1}^{2} \beta_{n}=2^{n} \beta^{\left(\begin{array}{c}
n+1 \\
2
\end{array}\right)} .
$$

This completes the proof of the theorem.

As a direct corollary of the Theorem 9 we conclude that the Hankel transform of the sequence of central trinomial coefficients $\left(t_{n}(1,1)\right)_{n \in \mathbb{N}_{0}}$ is $\mathcal{H}\left(\left(t_{n}(1,1)\right)_{n \in \mathbb{N}_{0}}\right)=\left(2^{n}\right)_{n \in \mathbb{N}_{0}}$. The same holds for the central binomial coefficients $\left(t_{n}(2,1)\right)_{n \in \mathbb{N}_{0}}$ and aerated central binomial coefficients $\left(t_{n}(0,1)\right)_{n \in \mathbb{N}_{0}}$.

Moreover, we see that Hankel transform of $t_{n}(\alpha, \beta)$ does not depend on value $\alpha$. That also confirms the well-known fact that Hankel transform $\mathcal{H}$ is not invertible.

\section{Coefficient array of polynomials $\left(P_{n}(x)\right)_{n \in \mathbb{N}_{0}}$ and factor- ization of the Hankel matrix}

In this section we deal with the coefficient array $\mathbf{C}(\alpha, \beta)=\left(a_{n, k}\right)_{n, k \in \mathbb{N}_{0}}$ of the polynomials $P_{n}(x)=\sum_{k=0}^{n} a_{n, k} x^{k}$ and make the factorization of the Hankel matrix $\mathbf{H}=\left(t_{i+j}\right)_{i, j \in \mathbb{N}_{0}}$. Note that $\mathbf{C}(\alpha, \beta)$ is a lower-triangular infinite matrix.

Example 3. First few terms of $\mathbf{C}(\alpha, \beta)$ are given by

$$
\mathbf{C}(\alpha, \beta)=\left(\begin{array}{ccccc}
1 & 0 & 0 & 0 & \ldots \\
-\alpha & 1 & 0 & 0 & \\
\alpha^{2}-2 \beta & -2 \alpha & 1 & 0 & \\
\alpha\left(3 \beta-\alpha^{2}\right) & 3\left(\alpha^{2}-\beta\right) & -3 \alpha & 1 & \\
\alpha^{4}-4 \alpha^{2} \beta+2 \beta^{2} & 4 \alpha\left(2 \beta-\alpha^{2}\right) & 2\left(3 \alpha^{2}-2 \beta\right) & -4 \alpha & \\
-\alpha\left(\alpha^{4}-5 \alpha^{2} \beta+5 \beta^{2}\right) & 5\left(\alpha^{4}-3 \alpha^{2} \beta+\beta^{2}\right) & 5 \alpha\left(3 \beta-2 \alpha^{2}\right) & 5\left(2 \alpha^{2}-\beta\right) & \\
\vdots & & & & \ddots
\end{array}\right) .
$$

Following theorem gives a nice representation of $\mathbf{C}(\alpha, \beta)$ in terms of Riordan arrays.

Theorem 10. Coefficient array $\mathbf{C}(\alpha, \beta)=\left(a_{n, k}\right)_{n, k \in \mathbb{N}_{0}}$ of polynomials $P_{n}(x)$ satisfies

$$
\mathbf{C}(\alpha, \beta)=\left(\frac{1-\beta x^{2}}{1+\alpha x+\beta x^{2}}, \frac{x}{1+\alpha x+\beta x^{2}}\right) .
$$

Proof. Denote by $f_{k}(x)$ the generating function of the sequence $\left(a_{n, k}\right)_{n \in \mathbb{N}_{0}}$, i.e.

$$
f_{k}(x)=\sum_{n=0}^{+\infty} a_{n, k} x^{n}, \quad(k \geq 0) .
$$

Our goal is to find $f_{0}(x)$ and to establish recurrence relation between $f_{k}(x)$ and $f_{k-1}(x)$. Let us rewrite (14) in the coefficient form, i.e.

$$
a_{n+1, j}-a_{n, j-1}+\alpha_{n} a_{n, j}+\beta_{n} a_{n-1, j}=0 \quad\left(n \in \mathbb{N}_{0}, j>0\right)
$$


and

$$
a_{n+1,0}+\alpha_{n} a_{n, 0}+\beta_{n} a_{n-1,0}=0 \quad\left(n \in \mathbb{N}_{0}\right) .
$$

Since $\alpha_{n}=\alpha$ and $\beta_{n}=\beta$ for $n \geq 2$, we multiply equation (17) by $x^{n}$, and sum over $n$ form 2 to $+\infty$. Hence we obtain

$$
\frac{f_{0}(x)-a_{0,0}-a_{1,0} x-a_{2,0} x^{2}}{x}=-\alpha\left(f_{0}(x)-a_{0,0}-a_{1,0} x\right)-\beta x\left(f_{0}(x)-a_{0,0}\right) .
$$

By exchanging $a_{0,0}=1, a_{1,0}=-\alpha$ and $a_{2,0}=\alpha^{2}-2 \beta$ in the previous equation we obtain

$$
f_{0}(x)=\frac{1-\beta x^{2}}{1+\alpha x+\beta x^{2}} .
$$

Similarly we multiply (16) by $x^{n}$ and sum over $n$ form 2 to $+\infty$. Hence we obtain

$$
\begin{aligned}
\frac{f_{j}(x)-a_{0, j}-a_{1, j} x-a_{2, j} x^{2}}{x}= & f_{j-1}(x)-a_{0, j-1}-a_{1, j-1} x \\
& -\alpha\left(f_{j}(x)-a_{0, j}-a_{1, j} x\right)-\beta x\left(f_{j}(x)-a_{0, j}\right) .
\end{aligned}
$$

Previous equation is equivalent to

$$
\begin{aligned}
\left(1+\alpha x+\beta x^{2}\right) f_{j}(x)= & x f_{j-1}(x)+a_{0, j}\left(1+\alpha x+\beta x^{2}\right)+a_{1, j}\left(x+\alpha x^{2}\right) \\
& +a_{2, j} x^{2}-a_{0, j-1} x-a_{1, j-1} x^{2} .
\end{aligned}
$$

Now by exchanging $a_{0, j}=0$ and

$$
a_{0, j-1}=a_{1, j}=\left\{\begin{array}{ll}
1, & j=1 \\
0, & j>1
\end{array}, \quad a_{2, j}=\left\{\begin{array}{ll}
-2 \alpha, & j=1 \\
1, & j=2, \\
0, & j>2
\end{array} \quad a_{1, j-1}= \begin{cases}-\alpha, & j=1 \\
1, & j=2 \\
0, & j>2\end{cases}\right.\right.
$$

to previous equation we obtain

$$
f_{j}(x)=\frac{x}{1+\alpha x+\beta x^{2}} f_{j-1}(x), \quad(j \geq 1) .
$$

Condition of the theorem directly follows from (18) and (19) and definition of Riordan array.

Riordan array $\mathbf{C}(\alpha, \beta)$ from (15) can be expressed as the following product

$$
\left(\frac{1-\beta x^{2}}{1+\alpha x+\beta x^{2}}, \frac{x}{1+\alpha x+\beta x^{2}}\right)=\left(\frac{1-\beta x^{2}}{1+\beta x^{2}}, \frac{x}{1+\beta x^{2}}\right) *\left(\frac{1}{1+\alpha x}, \frac{x}{1+\alpha x}\right) .
$$

Note that the first factor in $(20)$ is $\mathbf{C}(0, \beta)$, while the second factor is the $-\alpha$-th power of Binomial matrix $\mathbf{B}^{-\alpha}$. Hence we have $\mathbf{C}(\alpha, \beta)=\mathbf{C}(0, \beta) \mathbf{B}^{-\alpha}$. Moreover, $\mathbf{C}(0, \beta)=\left(M_{n, k}\right)_{n, k \in \mathbb{N}_{0}}$ is the coefficient array of the sequence of polynomials $\left((2 \sqrt{\beta})^{n} \hat{T}_{n}\left(\frac{x}{2 \sqrt{\beta}}\right)\right)_{n \in \mathbb{N}_{0}}$.

We obtain the closed-form expression for the general term $a_{n, k}$ starting from the well-known relation concerning Chebyshev polynomials

$$
\hat{T}_{n}(x)=n \sum_{k=0}^{\left\lfloor\frac{n}{2}\right\rfloor} \frac{(-1)^{k}(n-k-1) !}{2^{2 k} k !(n-2 k) !} x^{n-2 k} .
$$

From the previous relation we have

$$
(2 \sqrt{\beta})^{n} \hat{T}_{n}\left(\frac{x}{2 \sqrt{\beta}}\right)=n \sum_{k=0}^{\left\lfloor\frac{n}{2}\right\rfloor} \frac{(-1)^{k}}{n-k}\left(\begin{array}{c}
n-k \\
n-2 k
\end{array}\right) \beta^{k} x^{n-2 k} .
$$


and hence

$$
M_{n, j}= \begin{cases}\frac{2(-\beta)^{(n-j) / 2}}{n+j}\left(\begin{array}{ll}
\frac{n+j}{2} \\
\frac{n-j}{2}
\end{array}\right), & n-j \in 2 \mathbb{N} \\
0, & n-j \in 2 \mathbb{N}+1\end{cases}
$$

Now from (20) we obtain

$$
a_{n, k}=\sum_{j=0}^{n} M_{n, j}(-\alpha)^{j-k}\left(\begin{array}{l}
j \\
k
\end{array}\right)=\sum_{l=0}^{\left\lfloor\frac{n}{2}\right\rfloor} M_{n, n-2 l}(-\alpha)^{n-2 l-k}\left(\begin{array}{c}
n-2 l \\
k
\end{array}\right) .
$$

Replacing (21) in the previous relation yields to the required closed-form expression

$$
a_{n, k}=\sum_{l=0}^{\left\lfloor\frac{n}{2}\right\rfloor} \frac{(-1)^{n-k-l}}{n-l}\left(\begin{array}{c}
n-l \\
n-2 l
\end{array}\right)\left(\begin{array}{c}
n-2 l \\
k
\end{array}\right) \alpha^{n-2 l-k} \beta^{l} .
$$

Denote by $\mathbf{L}(\alpha, \beta)$ inverse matrix of $\mathbf{C}(\alpha, \beta)$, i.e. let

$$
\begin{aligned}
\mathbf{L}(\alpha, \beta) & =\mathbf{C}^{-1}(\alpha, \beta)=\left(\frac{1-\beta x^{2}}{1+\alpha x+\beta x^{2}}, \frac{x}{1+\alpha x+\beta x^{2}}\right)^{-1} \\
& =\left(\frac{1}{\sqrt{1-2 \alpha x+\left(\alpha^{2}-4 \beta\right) x^{2}}}, \frac{1-\alpha x-\sqrt{1-2 \alpha x+\left(\alpha^{2}-4 \beta\right) x^{2}}}{2 \beta x}\right) .
\end{aligned}
$$

Following theorem provides the factorization of the Hankel matrix $\mathbf{H}(\alpha, \beta)=\left(t_{i+j}(\alpha, \beta)\right)_{i, j \in \mathbb{N}_{0}}$.

Theorem 11. There holds

$$
\mathbf{H}(\alpha, \beta)=\mathbf{L}(\alpha, \beta) \mathbf{D}(\alpha, \beta) \mathbf{L}(\alpha, \beta)^{T},
$$

where $\mathbf{D}(\alpha, \beta)=\operatorname{diag}\left(d_{0}, d_{1}, d_{2}, \ldots\right)$ and $d_{0}=1, d_{i}=2 \beta^{i}$ for $i \in \mathbb{N}$.

Proof. Denote by $d_{i, j}$ the scalar product of polynomials $P_{i}(x)$ and $P_{j}(x)$ with respect to the weight $w(x)$. Due to the orthogonality, there holds $d_{i, j}=0$ for $i \neq j, d_{0,0}=d_{0}=\beta_{0}=1$ and $d_{i, i}=d_{i}=\beta_{0} \beta_{1} \cdots \beta_{i}=2 \beta^{i}$. On the other side, there holds

$$
\begin{aligned}
d_{i, j} & =\int_{-\infty}^{+\infty} P_{i}(x) P_{j}(x) w(x) d x=\int_{-\infty}^{+\infty}\left(\sum_{k=0}^{i} a_{i, k} x^{k}\right)\left(\sum_{l=0}^{j} a_{j, l} x^{l}\right) w(x) d x \\
& =\sum_{k=0}^{i} \sum_{l=0}^{j} a_{i, k} a_{j, l} \int_{-\infty}^{+\infty} x^{k+l} w(x) d x=\sum_{k=0}^{i} \sum_{l=0}^{j} a_{i, k} t(\alpha, \beta)^{k+l} a_{j, l}
\end{aligned}
$$

Let $\mathbf{D}(\alpha, \beta)=\left(d_{i, j}\right)_{i, j \in \mathbb{N}}=\operatorname{diag}\left(d_{0}, d_{1}, d_{2}, \ldots\right)$. Previous expression is equivalent with

$$
\mathbf{D}(\alpha, \beta)=\mathbf{C}(\alpha, \beta) \mathbf{H}(\alpha, \beta) \mathbf{C}(\alpha, \beta)^{T} .
$$

Finally, we obtain (24) by multiplying (25) by $\mathbf{L}(\alpha, \beta)$ and $\mathbf{L}(\alpha, \beta)^{T}$ from left and right side respectively.

For $i \neq j$, there holds $d_{i, j}=0$, due to the orthogonality of the polynomials $P_{i}(x)$ and $P_{j}(x)$ with repsect to the weight $w(x)$. 


\section{On the row sums of $\mathbf{L}(\alpha, \beta)=\left(\left(a_{n, k}\right)_{n, k \in \mathbb{N}_{0}}\right)^{-1}$}

In this section, we will be interested in the row sums $s_{n}(\alpha, \beta)=\sum_{k=0}^{n} l_{n, k}$ of the matrix $\mathbf{L}(\alpha, \beta)$ where

$$
\begin{aligned}
\mathbf{L}(\alpha, \beta) & =\mathbf{C}^{-1}(\alpha, \beta)=\left(\frac{1-\beta x^{2}}{1+\alpha x+\beta x^{2}}, \frac{x}{1+\alpha x+\beta x^{2}}\right)^{-1} \\
& =\left(\frac{1}{\sqrt{1-2 \alpha x+\left(\alpha^{2}-4 \beta\right) x^{2}}}, \frac{1-\alpha x-\sqrt{1-2 \alpha x+\left(\alpha^{2}-4 \beta\right) x^{2}}}{2 \beta x}\right) .
\end{aligned}
$$

That is the sequence A110181. The summation is taken from 0 to $n$ since $\mathbf{L}(\alpha, \beta)$ is lowertriangular matrix.

Example 4. The first few terms of the matrix $\mathbf{L}(\alpha, \beta)$ are given by

$$
\mathbf{L}(\alpha, \beta)=\left(\begin{array}{cccccc}
1 & 0 & 0 & 0 & 0 & \cdots \\
\alpha & 1 & 0 & 0 & 0 & \\
\alpha^{2}+2 \beta & 2 \alpha & 1 & 0 & 0 & \\
\alpha^{3}+6 \alpha \beta & 3\left(\alpha^{2}+\beta\right) & 3 \alpha & 1 & 0 & \\
\alpha^{4}+12 \alpha^{2} \beta+6 \beta^{2} & 4\left(\alpha^{3}+3 \alpha \beta\right) & 2\left(3 \alpha^{2}+2 \beta\right) & 4 \alpha & 1 & \\
\alpha^{5}+20 \alpha^{3} \beta+30 \alpha \beta^{2} & 5\left(\alpha^{4}+6 \alpha^{2} \beta+2 \beta^{2}\right) & 10\left(\alpha^{3}+2 \alpha \beta\right) & 5\left(2 \alpha^{2}+\beta\right) & 5 \alpha & \\
\vdots & & & & & \ddots
\end{array}\right)
$$

Recall that, according to Lemma 2, the generating function of the row sums of Riordan array $(g, f)$ is given by $\frac{g(x)}{1-f(x)}$. Hence, in our case, we obtain the following expression for the generating function of the row sums $s_{n}(\alpha, \beta)$ :

$$
S(x)=\frac{1}{2} \cdot \frac{1}{1-(\alpha+\beta+1) x}+\frac{1}{2} \cdot \frac{1-(\alpha+2 \beta) x}{1-(\alpha+\beta+1) x} \cdot \frac{1}{\sqrt{1-2 \alpha x+\left(\alpha^{2}-4 \beta\right) x^{2}}} .
$$

Example 5. First few terms of the sequence $\left(s_{n}(\alpha, \beta)\right)_{n \in \mathbb{N}_{0}}$ are given by

$$
\begin{aligned}
& s_{0}(\alpha, \beta)=1 \\
& s_{1}(\alpha, \beta)=\alpha+1 \\
& s_{2}(\alpha, \beta)=2 \beta+(\alpha+1)^{2}, \\
& s_{3}(\alpha, \beta)=3(2 \alpha+1) \beta+(\alpha+1)^{3}, \\
& s_{4}(\alpha, \beta)=4(3 \alpha(\alpha+1)+1) \beta+6 \beta^{2}+(\alpha+1)^{4},
\end{aligned}
$$

Let $V(x)=\frac{1-(\alpha+2 \beta) x}{1-(\alpha+\beta+1) x}$ and denote by $\left(v_{n}\right)_{n \in \mathbb{N}_{0}}(\alpha, \beta)$ the sequence with the generating function $V(x)$. By simple calculation we obtain

$$
v_{0}(\alpha, \beta)=1, \quad v_{n}(\alpha, \beta)=(\alpha+\beta+1)^{n-1}(1-\beta) \quad(n \in \mathbb{N}) .
$$

Now from the generating function $S(x)$ we can conclude that

$$
s_{n}(\alpha, \beta)=\frac{1}{2}(\alpha+\beta+1)^{n}+\frac{1}{2} \sum_{i=0}^{n} v_{i}(\alpha, \beta) t_{n-i}(\alpha, \beta) .
$$

Since both $v_{n}(\alpha, \beta)$ and $t_{n}(\alpha, \beta)$ are polynomials of $\alpha$ and $\beta$ for every $n \in \mathbb{N}_{0}$, the same holds for $s_{n}(\alpha, \beta)$. That fact will be used in later considerations.

We proceed to the evaluation of the Hankel transform $\left(h_{n}^{s}(\alpha, \beta)\right)_{n \in \mathbb{N}_{0}}=\mathcal{H}\left(\left(s_{n}(\alpha, \beta)\right)_{n \in \mathbb{N}_{0}}\right)$. The following theorem gives the closed-form expression for the weight function $w_{s}(x)$ whose moment sequence is $\left(s_{n}(\alpha, \beta)\right)_{n \in \mathbb{N}_{0}}$. 
Theorem 12. Sequence $\left(s_{n}(\alpha, \beta)\right)_{n \in \mathbb{N}_{0}}$ is the moment sequence of the weight function

$$
w_{s}(x)=\frac{\alpha+2 \beta-x}{2(\alpha+\beta+1-x)} w(x)=\left\{\begin{array}{ll}
\frac{\alpha+2 \beta-x}{2 \pi(\alpha+\beta+1-x) \sqrt{4 \beta-(x-\alpha)^{2}}}, & x \in[\alpha-2 \sqrt{\beta}, \alpha+2 \sqrt{\beta}] \\
0, & \text { otherwise }
\end{array} .\right.
$$

Proof. The proof is similar to the proof of Theorem 7 . Let

$$
P(z)=z^{-1} S\left(z^{-1}\right)=\frac{1}{2} \cdot \frac{1}{z-(\alpha+\beta+1)}+\frac{1}{2} \cdot \frac{z-(\alpha+2 \beta)}{z-(\alpha+\beta+1)} \cdot \frac{1}{\sqrt{(z-\alpha)^{2}-4 \beta}} .
$$

Denote by $x_{1,2}=\alpha \pm 2 \sqrt{\beta}$ the branch points of the square root in (27). We also take a regular branch of square root such that $\arg \left(z-x_{1}\right)=\arg \left(z-x_{2}\right)=0$ for $z \in\left(x_{2},+\infty\right)$. Selected branch is defined on $\mathbb{C} \backslash\left(x_{1}, x_{2}\right)$ and holds $\overline{P(z)}=P(\bar{z})$.

Direct evaluation yields to the following expression for the primitive function $P_{1}(z)$ of the $P(z)$ :

$$
\begin{aligned}
P_{1}(z)=\int P(z) d z= & \frac{1}{2} \log 4-\log (b-1)+\frac{1}{2} \log [z-\alpha+\rho(z)] \\
& +\frac{1}{2} \log [-a(b+1)-4 b+(b+1) z+(b-1) \rho(z)],
\end{aligned}
$$

where $\rho(z)=\sqrt{(z-\alpha)^{2}-4 \beta}$. We take a regular branch of $\log$ in the same way as in the Theorem 7. Note that the first two addends in previous expression are constant while third addend $l_{1}(z)=\frac{1}{2} \log [z-\alpha+\rho(z)]$ is of the same form as in Theorem 7 . Hence we have only to deal with the last addend $l_{2}(z)=\frac{1}{2} \log [-\alpha(\beta+1)-4 \beta+(\beta+1) z+(\beta-1) \rho(z)]$. Hence

$$
\lim _{y \rightarrow 0+} 2 \Im l_{2}(x+i y)= \begin{cases}\pi, & x<x_{1} \\ \pi+\arctan \left(\frac{(\beta-1) \sqrt{4 \beta-(x-\alpha)^{2}}}{-\alpha(1+\beta)-4 \beta+(\beta+1) x}\right), & x \in\left[x_{1}, \alpha+\frac{4 \beta}{\beta+1}\right) \\ \frac{\pi}{2}, & x=\alpha+\frac{4 \beta}{\beta+1} \\ \arctan \left(\frac{(b-1) \sqrt{4 \beta-(x-\alpha)^{2}}}{-\alpha(1+\beta)-4 \beta+(\beta+1) x}\right), & x \in\left(\alpha+\frac{4 \beta}{\beta+1}, x_{2}\right] \\ 0, & x>x_{2}\end{cases}
$$

Now by setting $\lambda(t)=-\frac{1}{\pi} \lim _{y \rightarrow 0+} \Im P_{1}(x+i y)$ and $w(t)=\lambda^{\prime}(t)$ we obtain the expression (29). This finishes the proof of the theorem.

According to the previous theorem we have the following integral representation of the sequence $\left(s_{n}(\alpha, \beta)\right)_{n \in \mathbb{N}_{0}}$ :

$$
s_{n}(\alpha, \beta)=\frac{1}{2 \pi} \int_{\alpha-2 \sqrt{\beta}}^{\alpha+2 \sqrt{\beta}} x^{n} \cdot \frac{\alpha+2 \beta-x}{1+\alpha+\beta-x} \cdot \frac{1}{\sqrt{4 \beta-(x-\alpha)^{2}}} d x .
$$

Example 6. By direct evaluation we obtain the first few members of the Hankel transform of $\left(s_{n}(\alpha, \beta)\right)_{n \in \mathbb{N}_{0}}$ :

$$
\begin{aligned}
& h_{0}^{s}(\alpha, \beta)=1, \\
& h_{1}^{s}(\alpha, \beta)=2 \beta \\
& h_{2}^{s}(\alpha, \beta)=\beta^{2}(4 \beta-1), \\
& h_{3}^{s}(\alpha, \beta)=4 \beta^{5}(2 \beta-1), \\
& h_{4}^{s}(\alpha, \beta)=\beta^{8}\left(16 \beta^{2}-12 \beta+1\right),
\end{aligned}
$$


It can be noticed that $h_{n}^{s}(\alpha, \beta)$ does not have the product form. However, a slight modification of the method based on orthogonal polynomials can be applied in this case. That is given by Theorem 15. We use linear multiplier and linear divisor transformations of the weight function showed in the following two transformation lemmas (see Gautschi [6]).

Lemma 13. [6] Consider the same notation as in Lemma 8 and let $\tilde{w}(x)=\frac{w(x)}{c-x}$ where $c>$ $\sup \operatorname{supp}(w)$. Consider the sequence $\left(r_{n}\right)_{n \geq-1}$ defined by

$$
r_{-1}=\int_{\mathbb{R}} \tilde{w}(x) d x, \quad r_{n}=c-\alpha_{n}-\frac{\beta_{n}}{r_{n-1}} \quad\left(n \in \mathbb{N}_{0}\right) .
$$

Then holds

$$
\begin{array}{lll}
\tilde{\alpha}_{0}=\alpha_{0}+r_{0}, & \tilde{\alpha}_{n}=\alpha_{n}+r_{n}-r_{n-1}, & \\
\tilde{\beta}_{0}=r_{-1}, & \tilde{\beta}_{n}=\beta_{n-1} \frac{r_{n-1}}{r_{n-2}} & (n \in \mathbb{N}) .
\end{array}
$$

Lemma 14. [6] Consider the same notation as in Lemma 8 and Lemma 13 and let $\tilde{w}(x)=$ $(d-x) w(x)$ where $d>\sup \operatorname{supp} w(x)$. Let the temporary sequences $\left(q_{n}\right)_{n \in \mathbb{N}_{0}}$ and $\left(e_{n}\right)_{n \in \mathbb{N}_{0}}$ be defined by

$$
q_{0}=\int_{\mathbb{R}} \tilde{w}(x) d x, \quad q_{n}=\alpha_{n-1}-e_{n-1}-d, \quad e_{0}=0, \quad e_{n}=\frac{\beta_{n}}{q_{n}} \quad(n \in \mathbb{N}) .
$$

Then holds

$$
\tilde{\alpha}_{n}=d+q_{n+1}+e_{n+1}\left(n \in \mathbb{N}_{0}\right), \quad \tilde{\beta}_{0}=r_{-1}, \quad \tilde{\beta}_{n}=q_{n+1} e_{n} \quad(n \in \mathbb{N}) .
$$

Theorem 15. Under the condition $\beta>1$, the Hankel transform $\left(h_{n}^{s}(\alpha, \beta)\right)_{n \in \mathbb{N}_{0}}$ of the row sums $\left(s_{n}(\alpha, \beta)\right)_{n \in \mathbb{N}_{0}}$ of $\mathbf{L}(\alpha, \beta)$ is given by:

$$
h_{n}^{s}(\alpha, \beta)=\beta^{\frac{n^{2}}{2}}\left[\frac{(\sqrt{\beta}-\sqrt{\beta-1})^{n}}{2-2 \sqrt{\beta(\beta-1)}-2 \beta}+\frac{(\sqrt{\beta}+\sqrt{\beta-1})^{n+1}}{2 \sqrt{\beta}}\right] .
$$

Proof. We again apply transformations of the weight function. Starting weight function is $w(x)=\left(\pi \sqrt{4 \beta-(x-\alpha)^{2}}\right)^{-1}$ and corresponding coefficients are given by (14):

$$
\alpha_{n}=\alpha \quad(n \in \mathbb{N}), \quad \beta_{0}=1, \quad \beta_{1}=2 \beta, \quad \beta_{n}=\beta \quad(n=2,3, \ldots) .
$$

The first transformation is $w^{(1)}(x)=\frac{w(x)}{\alpha+\beta+1-x}$. According to Lemma 13 we have to consider temporary sequence $\left(r_{n}\right)_{n \geq-1}$ defined by:

$$
r_{-1}=\int_{\mathbb{R}} w^{(1)}(x) d x, \quad r_{n}=\alpha+\beta+1-\alpha_{n}-\frac{\beta_{n}}{r_{n-1}} .
$$

By direct evaluation we find $\beta_{0}^{(1)}=r_{-1}=\frac{1}{\beta-1}$ for $\beta>1$. Moreover, using (36) and mathematical induction, we can prove

$$
r_{-1}=\frac{1}{\beta-1}, \quad r_{0}=2, \quad r_{n}=1, \quad(n \in \mathbb{N}) .
$$


Now according to Lemma 13 it holds

$$
\begin{aligned}
& \alpha_{0}^{(1)}=\alpha+2, \quad \alpha_{1}^{(1)}=\alpha-1, \quad \alpha_{n}^{(1)}=\alpha \quad(n \geq 2), \\
& \beta_{0}^{(1)}=\frac{1}{\beta-1}, \quad \beta_{1}^{(1)}=2(\beta-1), \quad \beta_{n}^{(1)}=\beta \quad(n \geq 2) .
\end{aligned}
$$

Second transformation is $w_{s}(x)=\frac{1}{2}(\alpha+2 \beta-x) w^{(1)}(x)$. According to Lemma 14, we have to consider temporary sequences $\left(q_{n}\right)_{n \in \mathbb{N}_{0}}$ and $\left(e_{n}\right)_{n \in \mathbb{N}_{0}}$ defined by

$$
e_{0}=0, \quad q_{0}=\int_{\mathbb{R}} w_{s}(x) d x, \quad q_{n}=\alpha_{n-1}-e_{n-1}-\alpha-2 \beta, \quad e_{n}=\frac{\beta_{n}}{q_{n}}, \quad(n \in \mathbb{N}) .
$$

According to (37) it holds

$$
q_{n}=-2 \beta-\frac{\beta}{q_{n-1}}, \quad(n \geq 3) .
$$

Using $q_{0}=s_{0}(\alpha, \beta)=1$ and previous expression we obtain $q_{1}=2(1-\beta), q_{2}=-2 \beta, q_{3}=\frac{1}{2}-2 \beta$, $e_{1}=-1$ and $e_{2}=-\frac{1}{2}$. Moreover, $\beta_{1}^{s}=q_{2} e_{1}=2 \beta$ and $\beta_{2}^{s}=q_{3} e_{2}=\frac{4 \beta-1}{4}$.

Since we are not able to guess a nice solution of the recursive equation (38) with initial value $q_{2}=-2 \beta$, we have to use another approach. According to the Heilermann formula (5) there holds

$$
\frac{h_{n+1}^{s}(\alpha, \beta)}{h_{n}^{s}(\alpha, \beta)}=s_{0}(\alpha, \beta) \cdot \beta_{1}^{s} \beta_{2}^{s} \cdots \beta_{n+1}^{s} \text {. }
$$

By replacing $s_{0}(\alpha, \beta)=1, \beta_{1}^{s}=2 \beta$ and $\beta_{i}^{s}=\beta_{i}^{(1)} q_{i+1} / q_{i}$ (Lemma 14) in the previous equation we obtain

$$
\frac{h_{n+1}^{s}(\alpha, \beta)}{h_{n}^{s}(\alpha, \beta)}=2 \beta \cdot \beta_{2}^{(1)} \frac{q_{3}}{q_{2}} \beta_{3}^{(1)} \frac{q_{4}}{q_{3}} \cdots \beta_{n+1}^{(1)} \frac{q_{n+2}}{q_{n+1}}=2 \beta^{n+1} \frac{q_{n+2}}{q_{2}}=-\beta^{n} q_{n+2},
$$

which implies

$$
q_{n}=-\frac{h_{n-1}^{s}(\alpha, \beta)}{\beta^{n-2} h_{n-2}^{s}(\alpha, \beta)} .
$$

Using the recurrence relation (38), we have

$$
-\frac{h_{n}^{s}(\alpha, \beta)}{\beta^{n-1} h_{n-1}^{s}(\alpha, \beta)}=-2 \beta+\frac{\beta}{\frac{h_{n-1}^{s}(\alpha, \beta)}{\beta^{n-2} h_{n-2}^{s}(\alpha, \beta)}} \quad(n \geq 3),
$$

and hence

$$
h_{n}^{s}(\alpha, \beta)=2 \beta^{n} h_{n-1}^{s}(\alpha, \beta)-\beta^{2(n-1)} h_{n-2}^{s}(\alpha, \beta) \quad(n \geq 3) .
$$

We introduce a new sequence $\left(u_{n}\right)_{n \in \mathbb{N}_{0}}$ defined by $u_{n}=h_{n}^{s}(\alpha, \beta) / \beta^{n^{2} / 2}$. Replacing into the previous equation yields

$$
u_{n}=2 \beta^{1 / 2} u_{n-1}-u_{n-2},(n \geq 3) .
$$

By solving the previous linear difference equation with the initial conditions $u_{0}=1$ and $u_{1}=$ $2 \sqrt{\beta}$, we obtain

$$
u_{n}=\frac{(\sqrt{\beta}-\sqrt{\beta-1})^{n}}{2-2 \sqrt{\beta(\beta-1)}-2 \beta}+\frac{(\sqrt{\beta}+\sqrt{\beta-1})^{n+1}}{2 \sqrt{\beta}} .
$$

Finally, by replacing $h_{n}^{s}(\alpha, \beta)=\beta^{\frac{n^{2}}{2}} u_{n}$ we finish the proof of the theorem. 
Theorem 15 gives the closed-form expression of the Hankel transform of $\left(s_{n}(\alpha, \beta)\right)_{n \in \mathbb{N}_{0}}$ under the condition $\beta>1$. We can extend this result to the general case. Recall that from (28) yields that $s_{n}(\alpha, \beta)$ is polynomial of $\alpha$ and $\beta$ for every $n$, and hence the same holds for the Hankel determinant $h_{n}^{s}(\alpha, \beta)$. Therefore, expressions obtained by expanding (35) and eliminating square root, hold for arbitrary $\beta$.

We end this section by the result concerning the generating function of the sequence $\left(u_{n}\right)_{n \in \mathbb{N}_{0}}$.

Theorem 16. The generating function $U(x)$ of the sequence $\left(u_{n}\right)_{n \in \mathbb{N}_{0}}$ is

$$
U(x)=\frac{1}{x^{2}-2 \sqrt{\beta} x+1} .
$$

Proof. The proof is similar to the proof of Theorem 10, using the recurrence relation (40) and initial values $u_{0}=1$ and $u_{1}=2 \sqrt{\beta}$.

Acknowledgement: The authors gratefully acknowledge the support from the research projects 144011 and 144023 of the Serbian Ministry of Science.

\section{References}

[1] Brualdi R., Kirkland S., Aztec diamonds and digraphs, and Hankel determinants of Schröder numbers, Journal of Combinatorial Theory, Series B 94 (2005) 334 - 351.

[2] Chihara T.S., An Introduction to Orthogonal Polynomials. Gordon and Breach, New York, 1978.

[3] Cvetković A.S., Rajković P.M., Ivković M., Catalan Numbers, the Hankel Transform and Fibonacci Numbers, Journal of Integer Sequences 5 (2002), Article 02.1.3.

[4] Eğecioğlu O., Redmond T., Ryavec C., Almost product evaluation of Hankel determinants The Electronic Journal of Combinatorics 15 (2008), \#R6.

[5] Eğecioğlu O., Redmond T., Ryavec C., A multilinear operator for almost product evaluation of Hankel determinants, Journal of Combinatorial Theory, Series A 117 (2010) 77-103.

[6] Gautschi W. Orthogonal Polynomials: Computation and Approximation, Clarendon Press - Oxford, 2003.

[7] Ismail M.E.H., Determinants with orthogonal polynomial entries, Journal of Computational and Applied Mathematics 178 (2005), 255-266.

[8] Junod A., Hankel determinants and orthogonal polynomials, Expo. Math. 21 (2003), 63-74.

[9] Krattenthaler C., Advanced determinant calculus, Seminaire Lotharingien Combin. 42 ("The Andrews Festschrift") (1999), Article B42q, 67 pp.

[10] Krattenthaler C., Advanced determinant calculus: A complement, Linear Algebra and its Applications 411 (2005) 68-166.

[11] Lang W., On sums of powers of zeros of polynomials, Journal of Comput. Appl. Mathematics, Vol. 89, Issue 2 (March 1998), 237-256. 
[12] Layman J.W., The Hankel Transform and Some of Its Properties, Journal of Integer Sequences 4 (2001), Article 01.1.5.

[13] Noe T.D., On the Divisibility of Generalized Central Trinomial Coefficients, Journal of Integer Sequences 9 (2006), Article 06.2,7.

[14] Rajković P.M, Petković M.D, Barry P., The Hankel Transform of the Sum of Consecutive Generalized Catalan Numbers, Integral Transforms and Special Functions 18 Issue 4 (2007) $285-296$.

[15] Shapiro L.W, Getu S., Woan W.J., Woodson L.C., The Riordan Group, Discr. Appl. Math. 34 (1991) 229-239.

[16] Sloane N.J.A., The On-Line Encyclopedia of Integer Sequences, Published electronically at http://www.research.att.com/ njas/sequences/, 2007.

[17] Sloane N.J.A., The On-Line Encyclopedia of Integer Sequences, Notices of the AMS 50 (2003), 912-915.

[18] Sprugnoli R., Riordan arrays and combinatorial sums, Discrete Math. 132 (1994), 267-290.

[19] Xin G., Proof of the Somos-4 Hankel determinants conjecture, Advances in Applied Mathematics 42 (2009), 152-156.

[20] Weisstein E.W., http://mathworld.wolfram.com/BinomialTransform.html/, 2007. 